\title{
Multi-Constrained Inverse Kinematics for the Human Hand
}

\author{
Ali-Akbar Samadani ${ }^{1}$, Dana Kulić ${ }^{1}$ and Rob Gorbet ${ }^{2}$
}

\begin{abstract}
Measuring the spatial and temporal characteristics of hand movement is a challenging task due to the large number of degrees of freedom (DOF) in the hand. This paper presents a multi-constrained inverse kinematics (IK) approach for hand motion estimation from motion capture data. The IK approach satisfies a set of prioritized motion and postural constraints for each hand joint and link. The high-priority constraint is fully satisfied, while the fulfilment of the lowpriority constraints is achieved as long as no conflict with the high-priority constraint exists. The proposed approach can aid marker-based motion capture technologies in accurately reconstructing discontinuities or erroneous marker trajectory segments resulting from occluded, missing, or flipped markers. The performance of the multi-constrained IK approach for the hand is tested for a full range of continuous hand motion.
\end{abstract}

\section{INTRODUCTION}

Fine hand finger tracking finds application in a wide range of fields including robotics, computer animation, ergonomics and rehabilitation (e.g.,[1]). A popular technology for measuring human movement is optical motion capture, in which reflective markers are secured to landmarks on the body and tracked using high speed cameras [2]. Optical motion capture systems have been used in clinical research as a tool to measure abnormalities in finger motions due to pathological conditions or neurological impairments (e.g., [3], [4]). However, accurately measuring fine hand and finger motions using optical motion capture is challenging due to issues of occlusion and marker flipping (misidentification of markers when two markers come into close proximity) caused by the large number of markers attached to a small area. Postprocessing of the motion capture data to correct flipped markers and apply spline fits to fill-in occluded markers is a tedious task as it requires retracing the marker trajectories frame by frame for discontinuities and unrealistic behaviours (due to marker flipping). Therefore, there is a need for a tool that can automatically reconstruct the defective motion segments.

Commercial motion capture systems (e.g., [2]) provide tools such as a reference kinematic model (skeleton) and virtual markers to help acquire clean and continuous motion data. Missing marker trajectories are recovered from neighbouring marker trajectories located on the same rigid body segment using inter-marker distance constraints in [5]. Interpolation techniques are used for reconstructing short missing marker trajectories (e.g.,[6]). The interpolation techniques

\footnotetext{
${ }^{1}$ A.A Samadani and ${ }^{1}$ D. Kulić are with the Electrical and Computer Engineering Department at the University of Waterloo, Waterloo, ON N2L 3G1 CANADA (e-mail: asamadani@uwaterloo.ca, dkulic@uwaterloo.ca).

${ }^{2} \mathrm{R}$. Gorbet is with the Centre for Knowledge Integration at the University of Waterloo, Waterloo, ON N2L 3G1 CANADA (e-mail: rbgorbet@uwaterloo.ca)
}

require data samples before and after the occlusion; hence, they are only applicable at the post-processing stage. In [7], an extrapolation approach for reconstructing short missing marker trajectory segments from the previously observed marker positions is presented with the assumption that the underlying human motions are linear or circular. Human motions are modeled using a conditional restricted Boltzmann machine in [8], and the resulting models are used to recover the missing marker positions during motion capture. In [9], a dynamic Bayesian network is used to reconstruct the missing marker trajectories assuming that the trajectories are smooth and that there is a correlation between different marker trajectories. Motion sequences are modeled using principal component analysis (PCA) as a hierarchy of linear models, and then a motion sequence with discontinuous marker trajectories is recovered through least squares optimization using available marker positions and the closest linear model representing the motion [10]. These approaches are datadriven; hence, their performance depends on the richness of the human motion data used for the model training. Another approach is the use of filtering methods, such as Unscented Kalman filtering [11]. This approach uses the velocity and acceleration of the tracked marker as the observation states along with those of the neighbouring markers to enhance the reconstruction accuracy. An inverse kinematic solver is then applied to ensure constant link length. The velocity and acceleration information can be very noisy if derived from the captured marker positions. Additional equipment can be used to directly measure velocity and acceleration, however this results in difficulties such as synchronization between separate measurement systems. Furthermore, this approach requires the placement of three markers with constant intermarker distances on each rigid body segment (in our case, the small area of the finger phalanges), which may result in increased marker occlusion and/or flipping.

Another class of approaches to determine the joint configuration given a measured posture or end-point position is Inverse Kinematics (IK). There are two main approaches in IK: analytical and differential. Analytical IK is obtained by finding a closed form solution for the inverse of the forward kinematic function, and is specific to a particular structure being studied. However, a closed-form solution is not guaranteed to exist for complex kinematic structures. In contrast with the analytical approach, the differential approach is applicable to any kinematic structure. Differential IK linearly maps the Cartesian velocity of a point along a kinematic chain to the joint velocities along the chain using the structure Jacobian. Comparisons between different IK approaches for articulated body motions can be found in [12] 
and [13].

A challenging problem in computing IK arises when dealing with kinematically complex structures (i.e., structures with a large number of DOF and kinematic constraints such as limitations in the joint range of motion). Furthermore, there might be scenarios where multiple and sometimes conflicting postural and motion constraints are specified in the operational space. A closed-form IK solution for computing finger joint angles from motion capture is reported in [14]. An iterative forward-backward IK is proposed in [15] that allows incorporating kinematic constraints for producing human-like animated motions. These methods are either computationally expensive; hence not applicable in real time, or/and are not designed to address deficiencies in the captured data resulting from missing, occluded or flipping markers. Furthermore, the application of the majority of the motion capture recovery techniques mentioned above is limited to scenarios where the position of the distal hand end-points (i.e., fingertips) is specified.

This paper adapts a multi-constrained IK approach proposed by Yamane and Nakamura [16] for estimating the hand joint configuration from motion capture data. The approach uses differential IK to solve for an appropriate joint configuration that best fits a specified link trajectory subject to a set of kinematic constraints. The multi-constrained IK allows for the specification of a Cartesian trajectory of any hand link as a high-priority constraint and reconstructs the motion of the hand joints to fit the specified trajectory. In order to comply with the kinematic constraints of the hand, the multi-constrained IK approach incorporates a set of low-priority motion and postural constraints in the form of reference link positions, joints with reference angles, and out of range joints. The high-priority constraint is satisfied first, with a solution for the low-priority constraints investigated in the null-space of the high-priority constraints (i.e., a space where joint angle modifications do not affect the specified trajectory of the high-priority constraint). In this study, a set of captured hand motion sequences is used to test the reconstruction accuracy and time resource requirements of the multi-constrained IK approach. The results demonstrate the capability of the multi-constrained IK to accurately and rapidly estimate the hand joint configuration given a high-priority Cartesian trajectory and a set of low-priority kinematic constraints.

\section{Methodology}

\section{A. Human Hand Model}

The hand is modeled as a rigid multi-body structure with multiple distal joints (end-effectors). Many robotic and animated human hand models have been proposed (e.g., [17], [18], [19], [20]). Typically, the rigid bodies (links) are connected via joints, which actuate the movement of the links. In this paper, a hand model with $26 \mathrm{DOF}$ is considered (Fig. 1) [21], but the proposed approach is applicable to any rigid body hand model. In the model shown in Fig. 1, there are 3 DOF at each of the wrist, thumb carpometacarpal (CMC) and metacarpophalangeal (MCP) joints. MCP joints

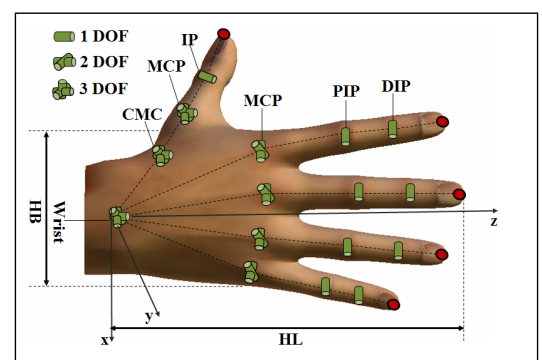

Fig. 1. Hand schematic showing DOF of hand joints. Finger tips are shown by red circles.

at the other four fingers of the hand (index, middle, ring and small (IMRS)), have 2 DOF each and their proximal interphalangeal (PIP) and distal interphalangeal (DIP) joints are 1 DOF. The thumb interphalangeal (IP) joint also has 1 DOF. The length of the hand links (fingers' phalanges) can be measured or estimated using two anthropomorphic parameters, hand length (HL) and hand breadth (HB), as described in [22] and [23].

\section{B. Multi-constrained Inverse Kinematics}

The multi-constrained IK allows for the trajectory of any point on any of the hand links to be specified (not necessarily the distal links) and uses a Jacobian to obtain a linear transformation between the link velocity and the velocities of the structure's joint angles [16]. The Jacobian is a matrix of partial derivatives of a given link position $\left(r_{i}\right)$ with respect to the structure's joints $(\theta)$ (Equation 1). Orin and Schrader in [24] discuss how to calculate the Jacobian matrix entries for different representations of joints and multi-bodies.

$$
J=\frac{\partial r_{i}}{\partial \theta}
$$

There are two levels of kinematic constraints considered in the multi-constrained IK; high-priority and low-priority constraints. High-priority constraints are fully satisfied, while fulfilment of the low-priority constraints is implemented in the null-space of the Jacobian of the high-priority constraints. For example, if the thumb is modelled as a 5DoF kinematic chain and the position of the thumb fingertip is specified as the high-priority constraint, 3 of the thumb DoFs are used to satisfy the position constraint, while the remaining DoF can be used to satisfy the low priority constraints. The Jacobian based method automatically identifies a decomposition of the available DoFs which can be used to satisfy the null-space constraints based on the current posture of the kinematic chain. Furthermore, in case of conflict between the lowpriority constraints, least squares optimization is applied to find an optimal solution for a set of low-priority constraints. Suppose the joint angles of a kinematic chain are arranged in a vector $\theta$ and $r_{P}$ is the current position of the link with a specified trajectory (high-priority constraint). The Jacobian matrix $J_{P}$ maps the velocity of the link $\left(\dot{r}_{P}\right)$ to the joint velocities $(\dot{\theta})$ :

$$
\dot{r}_{P}=J_{P} \dot{\theta} .
$$

When the Jacobian matrix is square and non-singular, it can be inverted to obtain the changes in joint angles for a desired change in position of the link. However, when dealing 
with structures with a large number of DOF, the mapping between the operational space (Cartesian space) and joint space is not unique. In this case, the pseudo-inverse, $J_{P}^{\sharp}$, can be used (Equation 3) to generate one of the feasible solutions.

$$
J_{P}^{\sharp}=J_{P}^{T}\left(J_{P} J_{P}^{T}\right)^{-1} .
$$

The resulting pseudo-inverse is then used to compute the appropriate joint velocities for the link at current position $r_{P}$ to reach the desired position $r_{P}^{r e f}$ at a reference velocity of $\dot{r}_{P}^{r e f}$ :

$$
\begin{aligned}
\dot{\theta} & =J_{P}^{\sharp} \dot{r}_{P}^{d}+\left(I-J_{P}^{\sharp} J_{P}\right) y, \\
\dot{r}_{P}^{d} & =\dot{r}_{P}^{r e f}+K_{P}\left(r_{P}^{r e f}-r_{P}\right),
\end{aligned}
$$

where $I$ is an identity matrix, $y$ is an arbitrary vector, $K_{P}$ is a positive-definite gain matrix, and $\dot{r}_{P}^{d}$ is the desired velocity for the link. The high priority constraint is satisfied via the first term in Equation 4. If the structure is redundant with respect to the task, additional constraints can be satisfied with the DOFs not involved with satisfying the high priority constraints, these low priority constraints are satisfied via the second term in Equation 4. Three types of low-priority constraints are considered: reference link positions, reference joint angles and joint motion range limits. Suppose there are $N_{F}$ links with reference positions $r_{F i} ; i=\left[1,2, \ldots, N_{F}\right]$, $N_{D}$ joints with reference joint angles $\theta_{\mathbf{D}}$, and $N_{R}$ joints with out-of-range joint angles $\theta_{\mathbf{R}}$. Consider a vector $\mathbf{P}_{\mathbf{L}}$ of all the low-priority constraints.

$$
\mathbf{P}_{\mathbf{L}}=\left[\begin{array}{lllll}
r_{F 1}^{T} & r_{F 1}^{T} \ldots & r_{F N_{F}}^{T} \theta_{\mathbf{D}}^{T} \theta_{\mathbf{R}}^{T}
\end{array}\right],
$$

The Jacobian $J_{L}$ maps the $\dot{\mathbf{P}}_{\mathbf{L}}$ to the joint velocities $\dot{\theta}$; $\dot{\mathbf{P}_{\mathbf{L}}}=J_{L} \dot{\theta}$. To obtain $J_{L}$, the Jacobian matrices $J_{F i}$ relating individual link velocities $r_{F i}$ to $\dot{\theta}$ need to be computed; $r_{F i}=J_{F i} \dot{\theta}$. The velocities of the joints with reference angles $\left(\theta_{\mathbf{D}}\right)$ and out of range joints $\left(\theta_{\mathbf{R}}\right)$ are related to $\dot{\theta}$ as:

$$
\begin{gathered}
\dot{\theta_{\mathbf{D}}}=J_{D} \dot{\theta}, \\
\dot{\theta_{\mathbf{R}}}=J_{R} \dot{\theta},
\end{gathered}
$$

where $J_{D}$ is obtained as:

$$
J_{D}(i, j)= \begin{cases}1, & \text { if } \theta_{D}(i)=\theta(j) \\ 0, & \text { otherwise. }\end{cases}
$$

The matrix $J_{R}$ is obtained in a similar way as $J_{D}$. Next, $J_{L}$ is formed by concatenating $J_{F i}, J_{D}$, and $J_{R}$ as follows:

$$
J_{L}=\left(\begin{array}{lllll}
J_{F 1}^{T} & \ldots & J_{F N_{F}}^{T} & J_{D}^{T} & J_{R}^{T}
\end{array}\right)^{T} .
$$

The desired velocity of the low-priority constraints, $\dot{P}_{L}^{d}$, is then computed using a series of feedback laws to account for differences between low-priority constraints and their current values $\left(r_{F_{i}}, \theta_{D}, \theta_{R}\right)$ :

$$
\begin{aligned}
\dot{r}_{F i}^{d} & =K_{F}\left(r_{F i}^{r e f}-r_{F i}\right), \\
\dot{\theta}_{D}^{d} & =K_{D}\left(\theta_{D}^{r e f}-\theta_{D}\right), \\
\dot{\theta}_{R i}^{d} & =\left\{\begin{array}{l}
K_{R i}\left(\theta_{R i}^{\max }-\theta_{R i}\right), i f\left(\theta_{R i}(i)>\theta_{R i}^{\max }\right) \\
K_{R i}\left(\theta_{R i}^{\min }-\theta_{R i}\right), i f\left(\theta_{R i}(i)<\theta_{R i}^{\max }\right),
\end{array}\right.
\end{aligned}
$$

where the superscripts $d$ and ref indicate the desired and reference quantities, respectively. For instance, $\dot{\theta}_{D}^{d}$ is the desired velocity to compensate for the difference between the reference joint angles $\left(\theta_{D}^{r e f}\right)$ and the current values of the joint angles $\left(\theta_{D}\right) . \theta_{R i}^{\max }$ and $\theta_{R i}^{\min }$ are the limits of $\theta_{R i}$ 's motion range. $K_{F}$ and $K_{D}$ are positive definite matrices and $K_{R i}$ is a positive scalar. After computing $J_{L}$ and $\dot{P}_{L}^{d}$, the change in velocity due to the low-priority constraints is computed:

$$
\begin{aligned}
\Delta \dot{P}_{L} & =\dot{P}_{L}^{d}-J_{L}\left(J_{P}^{\sharp} \dot{r}_{P}^{d}\right) \\
& =J_{L}\left(I-J_{P}^{\sharp} J_{P}\right) y \\
& =S y .
\end{aligned}
$$

$S$ is likely to be singular due to the multiple and perhaps conflicting kinematic constraints when dealing with complex structures. Applying the pseudo-inverse produces large and infeasible velocities in the vicinity of the singularity. Therefore, to invert $S$ for obtaining $y$, a singularity robust (SR) matrix inversion is used. The use of the SR inverse ensures that the $S$ matrix can be inverted, but introduces a small error (Equation 17), which is then compensated using the feedback control law (Equation 5).

$$
S^{*}=S^{T}\left(S S^{T}+k I\right)^{-1},
$$

where $I$ is an identity matrix and $k$ is a weighting parameter that controls the exactness versus feasibility of the solution (exact joint velocities versus erroneous but feasible ones). Next, $y$ is obtained as:

$$
y=S^{*} \Delta \dot{P}_{L} .
$$

The resulting $y$ vector is substituted into Equation (4) to obtain $\dot{\theta}$, which is then integrated to get an appropriate joint configuration $\theta$.

\section{EXPERIMENTAL RESULTS}

To test the performance of the multi-constrained IK, a continuous hand motion sequence consisting of 5 repetitions of hand opening and closing was used. The hand motion was recorded using ShapeHand dataglove [21] and local Euler angles for all the hand joints were collected at approximately 84 frames per second (total of 3240 time frames). This motion data is used for two purposes in our experiment. In the absence of optical motion capture data, the Euler angles and the hand model described in Section II-A are used to compute Cartesian trajectories using forward kinematics. In order to demonstrate the IK algorithm's ability to handle the underconstrained problem, only a small subset of these Cartesian joint trajectories will form the reference trajectories for the IK algorithm. It should be emphasized that typically this Cartesian information would come from an optical motion tracking system, so that the dataglove is not a required component of our approach to IK. The second use of the measured Euler angles is as a validation of the IK algorithm: it provides ground truth for comparing the joint angles estimated using multi-constrained IK with the measured (actual) angles. This validation data would not be available with optical motion capture.

In the experimental setup, the high-priority constraints (the links with specified trajectories) are the finger tips. The joint motion ranges are shown in Table I. The rotation around the $\mathrm{Z}$-axis for the thumb CMC and MCP are added according to the thumb kinematic model used in ShapeHand dataglove; 
TABLE I

HAND JOINTS' MOTION RANGE IN DEGREES [21], [22] (COUNTER-CLOCKWISE ROTATION \CLOCKWISE ROTATION).

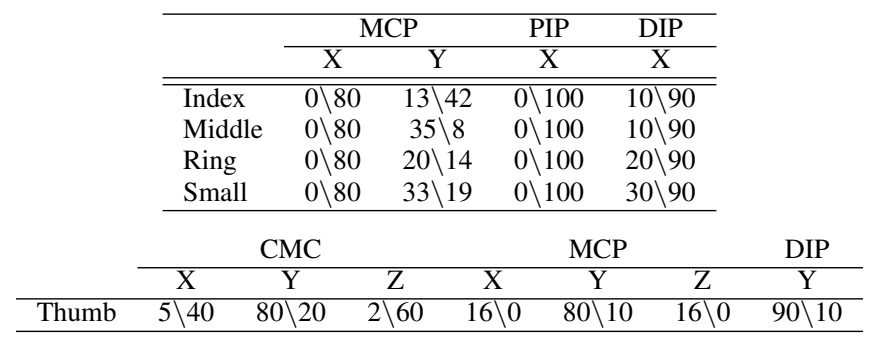

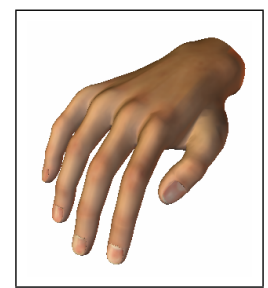

Fig. 2. The joint configuration, $\theta_{D}$, used as the reference hand posture.

hence, the motion range of the thumb joints is chosen to asymptotically accommodate the extrema of the thumb joint motion in the hand motion used here. Fig. 2 shows the joint configuration used as the reference posture. Furthermore, in the experimental setup, the reference high-priority velocity, $\dot{r}_{P}^{r e f}$, is set to zero as this quantity may not be available during on-line motion capture.

The performance of the multi-constrained IK is tested under two scenarios: the measured Cartesian trajectories of the DIP and PIP joints of the IMRS fingers (IP and MCP joints of the thumb) are used as the low-priority reference trajectory constraints in the first and second scenarios, respectively. In the implementation of the multi-constrained IK, at each time step, $r_{P}$ and $r_{F i}$, the current positions of the highpriority and low-priority link trajectories, are computed using forward kinematics from the latest estimated (by the multiconstrained IK) joint angles. Furthermore, at each time step, $r_{P}^{r e f}$ and $r_{F i}^{r e f}$, the reference position of the high-priority and low-priority link Cartesian trajectories, are updated with the values computed using the joint angles recorded by the dataglove. Note that the multi-constrained IK does not use any of the measured joint angles from the dataglove to compute other joint angles. The multi-constrained IK continuously monitors the hand joints for out of range joints to constrain them as described in Section II-B.

TABLE III

RMS ERRORS IN DEGREES BETWEEN THE ACTUAL AND ESTIMATED IMRS JOINT ANGLES IN THE TWO TESTING SCENARIOS: CONSTRAINED DIP AND CONSTRAINED PIP CARTESIAN POSITIONS.

\begin{tabular}{lcccccccc}
\hline & \multicolumn{4}{c}{ MCP } & \multicolumn{2}{c}{ PIP } & \multicolumn{2}{c}{ DIP } \\
\cline { 2 - 9 } Constraint & \multicolumn{2}{c}{ X } & \multicolumn{2}{c}{ Y } & \multicolumn{2}{c}{ X } & \multicolumn{2}{c}{ X } \\
\cline { 2 - 9 } DIP & PIP & DIP & PIP & DIP & PIP & DIP & PIP \\
\hline \hline Index & 0.26 & 0.25 & 0.09 & 0.07 & 0.79 & 0.90 & 0.35 & 2.62 \\
Middle & 0.36 & 0.28 & 0.09 & 0.04 & 1.08 & 0.59 & 0.45 & 2.52 \\
Ring & 0.55 & 0.35 & 0.07 & 0.04 & 1.48 & 0.88 & 0.57 & 3.36 \\
Small & 0.42 & 0.33 & 0.17 & 0.09 & 1.35 & 0.82 & 0.70 & 2.72 \\
\hline
\end{tabular}

The joint configuration for each timestep in the hand motion was then computed using the multi-constrained IK with the following parameters (chosen by trial and error); $k=$ $0.01 ; K_{P}=1.25 I_{3 \times 3} ; K_{R}=0.05 ; K_{F}=1.2 I_{3 \times 3} ; K_{D}=$ $0.01 I_{4 \times 4}$ (for thumb $K_{D}$ is a $7 \times 7$ matrix), where $I$ 's are identity matrices. The wrist was fixed in the hand motions used here. Fig. 3 shows an example joint angle trajectory obtained using the multi-constrained IK, compared to the actual joint angle data collected with the dataglove. Tables II and III show the root mean square (RMS) errors between the actual and estimated joint angle trajectories for each hand joint under the two testing scenarios. The low RMS errors, especially at DIP and IP joints in the case of constrained DIP positions for IMRS and constrained IP position for thumb, demonstrate the capability of the multi-constrained IK to accurately reconstruct the captured hand joint angle trajectories. As can be seen from the results (Fig. 3 and Tables II and III), the proper choice of the low-priority reference Cartesian trajectory constrain is an important factor for accurate estimation of the joint angle trajectories. In Fig. 3, in the case of constrained PIP position, there are large overshoots at the rising edges of the estimated joint angle trajectory. This might be either a result of inherent high-frequency artifacts in the captured motions or it is due to an abrupt change in the motion. By constraining the DIP position instead of PIP position, the overshooting behaviour is avoided and the joint angle trajectory is accurately estimated. This demonstrates the flexibility of the presented multi-constrained IK, which enables incorporating and replacing constraints at different points of the hand in order to construct realistic and accurate motion data. The resulting joint angle trajectories were also used to animate the hand motion and the reconstruction quality of the resulting hand motion was visually confirmed. The experiment was run using MATLAB and took $15.3 \mathrm{sec}$ (MATLAB timer) to obtain the IK solutions for 3240 time frames $(38 \mathrm{sec})$ of the hand motion used here. This shows the computational efficiency of the presented approach, which makes it suitable for on-line implementation with a motion capture system.

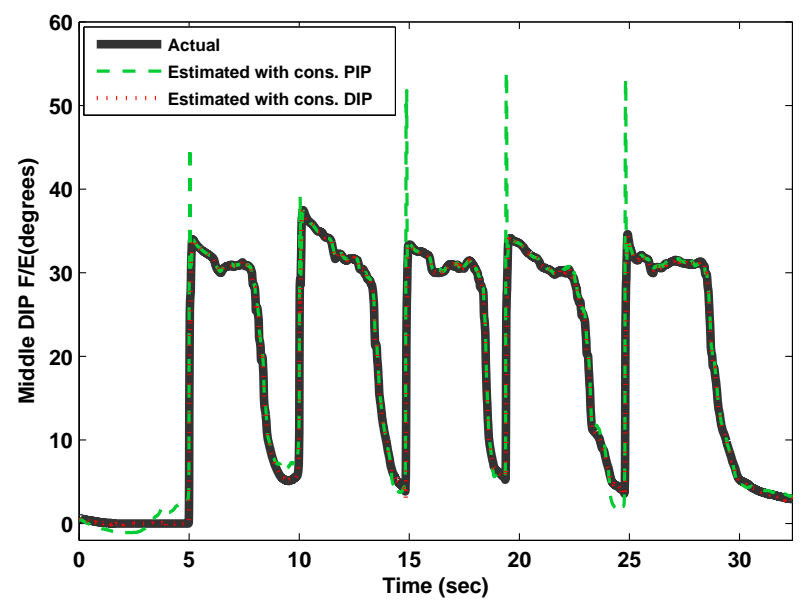

Fig. 3. The actual (bold black line) and estimated joint angle trajectories (with constrained PIP position: green dashed line; with constrained DIP position: red dotted line) for the middle's DIP flexion/extension. 
TABLE II

RMS ERRORS IN DEGREES BETWEEN THE ACTUAL AND ESTIMATED THUMB JOINT ANGLES IN THE TWO TESTING SCENARIOS: CONSTRAINED IP AND CONSTRAINED MCP CARTESIAN POSITIONS.

\begin{tabular}{|c|c|c|c|c|c|c|c|c|c|c|c|c|c|c|}
\hline \multirow[b]{3}{*}{ Constraint } & \multicolumn{6}{|c|}{$\mathrm{CMC}$} & \multicolumn{6}{|c|}{ MCP } & \multicolumn{2}{|c|}{ IP } \\
\hline & & & & & & & & & & & & & & \\
\hline & IP & MCP & IP & MCP & IP & MCP & IP & MCP & IP & MCP & IP & MCP & IP & MCP \\
\hline Thumb & 6.92 & 5.54 & 2.56 & 1.96 & 7.31 & 6.46 & 5.55 & 3.88 & 2.37 & 1.64 & 5.62 & 5.63 & 1.12 & 3.31 \\
\hline
\end{tabular}

\section{DisCUSSION AND CONCLUSIONS}

A multi-constrained IK approach was proposed to estimate joint angles given position data for a complex hand model. The performance of the multi-constrained IK solver for the hand is promising and shows the capability of the approach to accurately and rapidly estimate an appropriate joint configuration for a given hand pose. The multi-constrained IK is not limited to a specific structure and can be extended to other structures with varied number of DOF and kinematic constraints. An important feature of the multi-constrained IK approach is that it enables the specification of any point of interest on the hand as the high-priority constraint and reconstructs its motion with the highest accuracy. It also allows for the definition of low-priority constraints on link positions, joint angle errors, and joint motion ranges for any link and joint on the hand to enhance the reconstruction accuracy. Therefore, the multi-constrained IK is particularly suitable for reconstructing captured motions (which may have some missing marker data) by specifying the measured trajectory of a visible marker as the high-priority constraint and estimating a feasible hand joint configuration to fit the specified trajectory. The resulting joint configuration can then be used to construct the missing marker Cartesian trajectories by applying forward kinematics. Furthermore, The multiconstrained IK is fast; hence applicable for on-line motion capture.

The poorer estimation of the thumb joint angles (larger RMS value in Table II) as compared with the estimated IMRS joint angles (Table III) is due to inaccuracies in the thumb kinematic model. In general, the reconstruction performance of the multi-constrained IK depends on (ordered based on their importance): 1) the accuracy of the kinematic model, 2) the duration of the missing distal marker trajectory; performance deteriorates as the distal marker along a kinematic chain stays unseen for a long time, 3) the proper choice of the gain parameters. In future work, a method for optimizing the gain parameters will be investigated.

\section{REFERENCES}

[1] V. Pavlovic, R. Sharma, and T. Huang, "Visual interpretation of hand gestures for human-computer interaction: A review," Pattern Analysis and Machine Intelligence, IEEE Transactions on, vol. 19, no. 7, pp. 677-695, 1997.

[2] MotionAnalysis. [Online]. Available: www.motionanalysis.com

[3] C. Metcalf and S. Notley, "Modified kinematic technique for measuring pathological hyperextension and hypermobility of the interphalangeal joints," Biomedical Engineering, IEEE Transactions on, no. 99 , pp. 1224-1231, 2011.

[4] R. Degeorges, J. Parasie, D. Mitton, N. Imbert, J. Goubier, and F. Lavaste, "Three-dimensional rotations of human three-joint fingers: an optoelectronic measurement. preliminary results," Surgical and Radiologic Anatomy, vol. 27, no. 1, pp. 43-50, 2005.
[5] A. Hornung, S. Sar-Dessai, and L. Kobbelt, "Self-calibrating optical motion tracking for articulated bodies," in Virtual Reality, 2005. Proceedings. VR 2005. IEEE. IEEE, 2005, pp. 75-82.

[6] D. Wiley and J. Hahn, "Interpolation synthesis of articulated figure motion," Computer Graphics and Applications, IEEE, vol. 17, no. 6 , pp. 39-45, 1997.

[7] T. Piazza, J. Lundström, A. Hugestrand, A. Kunz, and M. Fjeld, "Towards solving the missing marker problem in realtime motion capture," in Proceedings of the International Design Engineering Technical Conference, 2009.

[8] G. Taylor, G. Hinton, and S. Roweis, "Modeling human motion using binary latent variables," Advances in neural information processing systems, vol. 19, pp. 1345-1352, 2007.

[9] L. Li, J. McCann, N. Pollard, and C. Faloutsos, "Dynammo: Mining and summarization of coevolving sequences with missing values," in Proceedings of the 15th ACM SIGKDD international conference on Knowledge discovery and data mining. ACM, 2009, pp. 507-516.

[10] G. Liu and L. McMillan, "Estimation of missing markers in human motion capture," The Visual Computer, vol. 22, no. 9, pp. 721-728, 2006

[11] A. Aristidou and J. Lasenby, "Real-time marker prediction and cor estimation in optical motion capture," The Visual Computer, pp. 1-20, 2012

[12] L. Unzueta, M. Peinado, R. Boulic, and Á. Suescun, "Full-body performance animation with sequential inverse kinematics," Graphical Models, vol. 70, no. 5, pp. 87-104, 2008.

[13] A. Aristidou and J. Lasenby, "Inverse kinematics: A review of existing techniques and introduction of a new fast iterative solver," 2009.

[14] C. Lien and C. Huang, "Model-based articulated hand motion tracking for gesture recognition," Image and Vision Computing, vol. 16, no. 2, pp. 121-134, 1998.

[15] A. Aristidou and J. Lasenby, "Motion capture with constrained inverse kinematics for real-time hand tracking," in Communications, Control and Signal Processing (ISCCSP), 2010 4th International Symposium on. IEEE, 2010, pp. 1-5.

[16] K. Yamane and Y. Nakamura, "Natural motion animation through constraining and deconstraining at will," Visualization and Computer Graphics, IEEE Transactions on, vol. 9, no. 3, pp. 352-360, 2003.

[17] A. Miller, P. Allen, V. Santos, and F. Valero-Cuevas, "From robotic hands to human hands: a visualization and simulation engine for grasping research," Industrial Robot: An International Journal, vol. 32 , no. 1 , pp. 55-63, 2005

[18] N. Fukaya, S. Toyama, T. Asfour, and R. Dillmann, "Design of the tuat/karlsruhe humanoid hand," in Intelligent Robots and Systems, 2000.(IROS 2000). Proceedings. 2000 IEEE/RSJ International Conference on, vol. 3. IEEE, 2000, pp. 1754-1759.

[19] M. Cutkosky, "On grasp choice, grasp models, and the design of hands for manufacturing tasks," Robotics and Automation, IEEE Transactions on, vol. 5, no. 3, pp. 269-279, 1989.

[20] A. Caffaz and G. Cannata, "The design and development of the disthand dextrous gripper," in Robotics and Automation, 1998. Proceedings. 1998 IEEE International Conference on, vol. 3. IEEE, 1998, pp. $2075-2080$.

[21] Measurand, "Motion capture systems," 2009. [Online]. Available: http://www.measurand.com

[22] E. Pitarch, "Virtual human hand: Grasping strategy and simulation," PhD diss., Universitat Politècnica de Catalunya, Barcelona, Spain, 2007.

[23] B. Buchholz, J. Thomas, and A. Steven, "Anthropometric data for describing the kinematics of the human hand," Ergonomics, vol. 35, no. 3, pp. 261-273, 1992.

[24] D. Orin and W. Schrader, "Efficient computation of the jacobian for robot manipulators," The International Journal of Robotics Research, vol. 3, no. 4, pp. 66-75, 1984. 